\title{
BIOADSORCIÓN DE PLOMO (II) PRESENTE EN SOLUCIÓN ACUOSA SOBRE RESIDUOS DE FIBRAS NATURALES PROCEDENTES DE LA INDUSTRIA IXTLERA (Agave lechuguilla Torr. Y Yucca carnerosana (Trel.) McKelvey)
}

\author{
Nahum Andrés MEDELLÍN-CASTILLO ${ }^{1 *}$, Miriam Giovana HERNÁNDEZ-RAMÍREZ ${ }^{1}$, \\ Jacob Josafat SALAZAR-RÁBAGO ${ }^{2}$, Gladis Judith LABRADA-DELGADO ${ }^{3}$ y Antonio ARAGÓN-PIÑA ${ }^{4}$
}

${ }^{1}$ Facultad de Ingeniería, Centro de Investigación y Estudios de Posgrado, Universidad Autónoma de San Luis Potosí. Avenida Dr. Manuel Nava 8, San Luis Potosí, San Luis Potosí, México, C.P. 78290

${ }^{2}$ Facultad de Ciencias Químicas, Centro de Investigación y Estudios de Posgrado, Universidad Autónoma de San Luis Potosí, Avenida Dr. Manuel Nava 8, San Luis Potosí, San Luis Potosí, México, C.P. 78290

${ }^{3}$ Instituto Potosino de Investigación Científica y Tecnológica. Camino a la Presa de San José 2055, San Luis Potosí, San Luis Potosí, México, C.P. 78216

${ }^{4}$ Instituto de Metalurgía, Universidad Autónoma de San Luis Potosí. Avenida Sierra Leona 550, San Luis Potosí, San Luis Potosí, México, C.P. 78210

*Autor para correspondencia: nahumanca@hotmail.com

(Recibido marzo 2016; aceptado agosto 2016)

Palabras clave: materiales lignocelulósicos, mecanismo de bioadsorción, sitios activos, microprecipitación

\section{RESUMEN}

El plomo ha sido reconocido como uno de los metales más tóxicos por su efecto negativo sobre el ambiente. En el presente trabajo se evaluó el uso potencial de residuos de Agave lechuguilla Torr. (lechuguilla) y Yucca carnerosana (Trel.) McKelvey (yuca) procedentes de la industria ixtlera para remover iones $\mathrm{Pb}$ (II) presentes en solución acuosa. Los datos del equilibrio de bioadsorción mostraron que al aumentar el $\mathrm{pH}$ de la solución de 2.0 a 5.0, la capacidad de bioadsorción de ambos materiales se incrementa. Este efecto se explicó considerando que la carga superficial negativa de estos materiales es mayor al aumentar el $\mathrm{pH}$ y por ello se favorece la remoción de $\mathrm{Pb}$ (II). El efecto de la temperatura de la solución en las isotermas de bioadsorción, expuso la naturaleza endotérmica del proceso. La energía libre de Gibbs y la entropía calculada $\left(\Delta \mathrm{G}^{\circ}\right.$ y $\left.\Delta \mathrm{S}^{\circ}\right)$ indicaron la espontaneidad de la bioadsorción y la afinidad del $\mathrm{Pb}$ (II) en solución por los bioadsorbentes, respectivamente. La variación de las capacidades de bioadsorción de la lechuguilla y la yuca se atribuyó a la diferencia en el contenido de sitios ácidos y lignina, lo que condujo a la presencia de diversos mecanismos de bioadsorción. En ambos materiales, la bioadsorción de $\mathrm{Pb}$ (II) ocurre por los mecanismos de interacciones $\pi$-catión, atracciones electrostáticas e intercambio iónico; además en el caso de la lechuguilla se evidenció un proceso de microprecipitación.

Key words: lignocellulosic materials, biosorption mechanism, active sites, microprecipitation

\begin{abstract}
Lead has been recognized as one of the most toxic metals due to its negative effect on the environment. In the present work, the potential use of Agave lechuguilla Torr.
\end{abstract}


(lechuguilla) and Yucca carnerosana (Trel.) McKelvey (yucca) residues from the ixtle industry to remove $\mathrm{Pb}$ (II) ions in an aqueous solution was evaluated. The adsorption equilibrium data showed that when increasing the $\mathrm{pH}$ in the solution from 2.0 to 5.0, the biosorption capacity of both materials increases. This effect might be explained by the negative surface charge of these materials, which is greater at high $\mathrm{pH}$, favoring the removal of $\mathrm{Pb}$ (II). The effect of the temperature of the solution on the biosorption isotherms revealed the endothermic nature of the biosorption process. The Gibbs free energy change and the calculated entropy $\left(\Delta \mathrm{G}^{\circ}\right.$ and $\left.\Delta \mathrm{S}^{\circ}\right)$ indicated the spontaneity of biosorption and the affinity of $\mathrm{Pb}$ (II) in the solution for biosorbents, respectively. The variation of the biosorption capacities of lechuguilla and yucca was attributed to the different content of acid sites and lignin, which resulted in the presence of different biosorption mechanisms. In both materials, the biosorption of $\mathrm{Pb}$ (II) occurs by $\pi$-cation interactions, electrostatic attractions and ion exchange; moreover, a microprecipitation process on lechuguilla was evinced.

\section{INTRODUCCIÓN}

La contaminación del agua por metales pesados se ha convertido en un tema de importancia debido a que representa un problema grave de salud pública (Demirbas 2008). El plomo (Pb) es conocido por ser uno de los metales pesados más tóxico, aún a bajas concentraciones, y que contamina los medios acuáticos (Blázquez et al. 2010, Taşar et al. 2014). Los efectos de la toxicidad del plomo en el ser humano incluyen encefalopatías, deterioro cognitivo, trastornos del comportamiento, daño renal, anemia y afectaciones en el sistema reproductivo (O'Connell et al. 2008).

Existen diversos métodos físicos y químicos para remover los metales pesados de soluciones acuosas, entre los que se encuentran la precipitación química, el intercambio iónico, la separación con membranas, la adsorción y los métodos electroquímicos (Reddad et al. 2002, Taşar et al. 2014). Normalmente, estos métodos involucran altos costos de operación o tienen eficiencias de remoción bajas que son insuficientes para cumplir con los requerimientos del proceso o la legislación ambiental (Reddad et al. 2002). Sin embargo, la bioadsorción es considerada como un proceso eficiente y de bajo costo para la remoción de metales pesados (Srivastava et al. 2015). Por lo general, la bioadsorción es considerada un tipo específico de adsorción basada en la capacidad de la biomasa o biomateriales para acumular agentes contaminantes en sus tejidos inertes. En los últimos años, se ha tomado interés por el uso de una gran cantidad de biomateriales como las plantas naturales y los residuos agrícolas e industriales ya que suelen ser abundantes, de bajo costo y no tóxicos ni peligrosos (Romero et al. 2010).

El Agave lechuguilla Torr. (lechuguilla) y la Yucca carnerosana (Trel.) McKelvey (yuca), son plantas suculentas comunes en las áreas semiáridas y áridas del centro y norte de México (zona ixtlera) y suroeste de los Estados Unidos (Sheldon 1980, Romero et al. 2005). Las fibras de Agave lechuguilla y fourcroydes (henequén) contienen un $80,15 \mathrm{y}$ $5 \% \mathrm{p} / \mathrm{p}$, aproximadamente de celulosa, lignina y hemicelulosa, respectivamente (Vieira et al. 2002), lo cual resulta favorable para la remoción de iones de metales pesados, ya que la celulosa, la pectina, la lignina y los taninos presentan diversos grupos funcionales que incrementan la remoción de estos contaminantes (Gupta et al. 2009).

Por otro lado, se han reportado diversos estudios sobre el uso de Agave lechuguilla Torr. como bioadsorbente para la remoción de $\mathrm{Cr}$ (III) y $\mathrm{Cr}$ (VI) en solución acuosa (Romero et al. 2005, Romero et al. 2006, Romero et al. 2009). En dichos estudios se han determinado parámetros termodinámicos y cinéticos del equilibrio de adsorción y se han propuesto modelos de transporte de masa para describir la adsorción de $\mathrm{Cr}$ (III) en continuo. Únicamente se han evaluado los efectos del $\mathrm{pH}$, la temperatura y la concentración inicial del metal en la solución sobre la remoción de $\mathrm{Pb}$ (II) en fibras de Agave americana (Hamissa et al. 2010), sin relacionarlos con algunas propiedades físicas, químicas y de textura, de los materiales y sin establecer un posible mecanismo de bioadsorción. En el caso de la Yucca carnerosana (Trel.) McKelvey no se encontró evidencia bibliográfica de que se haya estudiado su uso como potencial bioadsorbente. Además, no se han comparado las propiedades de la lechuguilla y la yuca y determinado la relación que estas tienen con la bioadsorción de $\mathrm{Pb}$ (II) en soluciones acuosas.

Por lo anterior, el objetivo de este trabajo es evaluar el equilibrio de bioadsorción de $\mathrm{Pb}$ (II) en soluciones acuosas sobre residuos de fibras naturales 
de Agave lechuguilla Torr. y Yucca carnerosana (Trel.) McKelvey de la industria ixtlera y de igual forma, relacionar las propiedades físicas, químicas y de textura, de estos biomateriales con el mecanismo de bioadsorción.

\section{MATERIALES Y MÉTODOS}

\section{Muestras de los bioadsorbentes}

Las muestras de Agave lechuguilla Torr. (lechuguilla) y Yucca carnerosana (Trel.) McKelvey (yuca) fueron proporcionadas por la empresa ixtlera Compañía Mexicana del Desierto de la ciudad de Matehuala, San Luis Potosí, México. Las muestras corresponden a residuos del proceso de la empresa cuyas cantidades generadas por semana son de alrededor de 1.5 toneladas.

\section{Preparación de los bioadsorbentes}

Las muestras proporcionadas fueron lavadas con agua destilada y una solución $0.01 \mathrm{M}$ de $\mathrm{HCl}$, enseguida se secaron los materiales en una estufa a $80^{\circ} \mathrm{C}$ durante $24 \mathrm{~h}$ y posteriormente fueron molidas y tamizadas a un diámetro de partícula promedio de $0.707 \mathrm{~mm}$.

\section{Determinación de propiedades físicas, químicas y de textura}

El área específica de ambos materiales se determinó por medio de un equipo de fisisorción, Micromeritics, modelo ASAP 2020.

Los sitios activos de las muestras de lechuguilla y yuca se determinaron por el método de titulación ácido-base propuesto por Boehm (1994).

La carga superficial de los bioadsorbentes y el punto de carga cero (PCC) se evaluó empleando un método de titulación potenciométrica descrito por Salazar y Leyva (2016).

El contenido de humedad y cenizas se determinó mediante métodos estándar gravimétricos (AOAC 1990).

Los espectros al infrarrojo de las muestras de lechuguilla y yuca libres y saturadas con $\mathrm{Pb}$ (II) se obtuvieron en un espectrofotómetro Thermo Scientific, modelo Nicolet iS10 usando la técnica de reflectancia total atenuada (ATR).

La estabilidad térmica de los bioadsorbentes se caracterizó empleando un Analizador termogravimétrico (TGA), Thermal Advantage, modelo TGA Q500. El intervalo de temperatura para el análisis de las muestras fue de los 20 a los $600{ }^{\circ} \mathrm{C}$, con una velocidad de calentamiento de $10{ }^{\circ} \mathrm{C} / \mathrm{min}$.
La textura superficial de los materiales bioadsorbentes se caracterizó empleando un microscopio electrónico de barrido ambiental (ESEM, por sus siglas en inglés), FEI, modelo Quanta 200. Este equipo cuenta con un detector para realizar análisis de espectroscopía de energía dispersiva de rayos-X (EDS, por sus siglas en inglés), EDAX, modelo DX4, que se utilizó para determinar la composición química superficial de los bioadsorbentes.

\section{Método para la obtención de datos experimentales de las isotermas de bioadsorción de $\mathbf{P b}$ (II)}

Los datos experimentales del equilibrio de bioadsorción se obtuvieron en un bioadsorbedor de lote de acuerdo con la siguiente metodología: a partir de una solución patrón de $\mathrm{Pb}$ (II) de $1000 \mathrm{mg} / \mathrm{L}$ se prepararon diversas soluciones de $\mathrm{Pb}$ (II) en matraces volumétricos de $50 \mathrm{~mL}$, con concentraciones que variaron de 20 a $400 \mathrm{mg} / \mathrm{L}$. Los matraces se aforaron con una solución amortiguadora de $\mathrm{pH}$ establecido en 2.0, 3.0 ó 5.0 que se obtuvo al mezclar volúmenes predeterminados de soluciones $0.01 \mathrm{~N}$ de $\mathrm{NaOH}$ y $\mathrm{HNO}_{3}$. Enseguida, se tomaron muestras iniciales de $10 \mathrm{~mL}$ para corroborar la concentración inicial de $\mathrm{Pb}$ (II). En viales cónicos de polietileno de $50 \mathrm{~mL}$, considerados como bioadsorbedores de lote, se agregó $0.2 \mathrm{~g}$ de bioadsorbente (lechuguilla o yuca) y los $40 \mathrm{~mL}$ de la solución de $\mathrm{Pb}$ (II) restante. Posteriormente, los bioadsorbedores de lote se colocaron en una rejilla y se sumergieron en un baño termostático con agua. El bioadsorbente se dejó en contacto con la solución de $\mathrm{Pb}$ (II) hasta alcanzar el equilibrio. En experimentos previos se determinó que cuatro días son adecuados para alcanzar el equilibrio de bioadsorción. Los bioadsorbedores se colocaron en la rejilla de un agitador orbital y se agitaron a $300 \mathrm{rpm}$ por 15 min tres veces al día; al alcanzar el equilibrio se tomó una muestra de $10 \mathrm{~mL}$ para determinar la concentración final de la solución. El pH de la solución se verificó durante el experimento y se ajustó usando soluciones $0.01 \mathrm{~N}$ de $\mathrm{NaOH}$ o $\mathrm{HNO}_{3}$. La capacidad de bioadsorción de $\mathrm{Pb}$ (II) de los materiales se calculó por medio de un balance de masa representado por la siguiente ecuación:

$\mathrm{q}=\frac{\mathrm{V}_{0}\left(\mathrm{C}_{0}-\mathrm{C}\right)}{\mathrm{m}}$

donde $\mathrm{C}$ es la concentración de $\mathrm{Pb}$ (II) en el equilibrio de bioadsorción $(\mathrm{mg} / \mathrm{L}) ; \mathrm{C}_{0}$ es la concentración inicial de $\mathrm{Pb}$ (II) en la solución del bioadsorbedor $(\mathrm{mg} / \mathrm{L}) ; \mathrm{m}$ es la masa de lechuguilla o yuca $(\mathrm{g})$; $\mathrm{q}$ es la capacidad de bioadsorción de $\mathrm{Pb}$ (II) de los materiales $(\mathrm{mg} / \mathrm{g})$ y $\mathrm{V}_{0}$ es el volumen inicial de $\mathrm{Pb}$ (II) en el bioadsorbedor (L). 
CUADRO I. PROPIEDADES FISICAS, QUÍMICAS Y DE TEXTURA DE LOS BIOADSORBENTES

\begin{tabular}{|c|c|c|c|c|c|c|c|c|c|}
\hline Bioadsorbente & $\begin{array}{c}\text { Sitios } \\
\text { lactónicos } \\
\text { (meq/g) }\end{array}$ & $\begin{array}{c}\text { Sitios } \\
\text { carboxílicos } \\
(\mathrm{meq} / \mathrm{g})\end{array}$ & $\begin{array}{c}\text { Sitios } \\
\text { fenólicos } \\
\text { (meq/g) }\end{array}$ & $\begin{array}{c}\text { Sitios } \\
\text { ácidos } \\
\text { totales (meq } / \mathrm{g} \text { ) }\end{array}$ & $\begin{array}{c}\text { Sitios } \\
\text { básicos } \\
\text { totales (meq/g) }\end{array}$ & $\begin{array}{c}\text { Área } \\
\text { específica } \\
\left(\mathrm{m}^{2} / \mathrm{g}\right)\end{array}$ & $\begin{array}{c}\text { Humedad } \\
\%\end{array}$ & $\begin{array}{c}\text { Cenizas } \\
\%\end{array}$ & PCC \\
\hline Lechuguilla & 0.058 & 0.0420 & 0.512 & 0.612 & 0.0 & 0.007 & 3.91 & 0.692 & 3.25 \\
\hline Yuca & 0.154 & 0.0001 & 0.528 & 0.682 & 0.0 & 0.098 & 4.28 & 0.501 & 3.00 \\
\hline
\end{tabular}

PCC $=$ Punto de carga cero

Las concentraciones de $\mathrm{Pb}$ (II) en la solución acuosa fueron determinadas por absorción atómica empleando un espectrofotómetro de doble haz, Varian, modelo SpectrAA-20.

\section{RESULTADOS Y DISCUSIÓN}

\section{Propiedades fisicas, químicas y de textura de la lechuguilla y la yuca}

Las propiedades fisicas, químicas y de textura de las muestras de lechuguilla y yuca se muestran en el cuadro I. Los resultados revelaron que ambos materiales son predominantemente de carácter ácido ya que no se lograron cuantificar sitios básicos en la superficie de las muestras analizadas. Por otra parte, se encontró que la concentración de los sitios ácidos es ligeramente mayor en las muestras de yuca. También se observó que la concentración de sitios ácidos en ambas muestras disminuye en el orden siguiente: fenólicos $>$ lactónicos $>$ carboxílicos. La predominancia de los sitios fenólicos frente a los otros tipos de grupos cuantificados (láctonicos y carboxílicos) se atribuye a la estructura molecular de la lignina, la cual presenta en abundancia este tipo de sitios activos. La importancia de este tipo de sitios radica en el hecho de que, al estar presentes en solución acuosa y dependiendo del $\mathrm{pH}$ de la misma, poseen la capacidad de ceder o aceptar protones, lo cual puede favorecer la captación de cationes metálicos en la solución (Salazar y Leyva 2016). Es importante mencionar que este es el primer trabajo donde se cuantifica la concentración de sitios activos de las muestras de lechuguilla y yuca.

Aunado a lo anterior, se determinó el PCC a partir de las distribuciones de carga superficial de la lechuguilla y la yuca que se muestran en la figura 1. El PCC de la lechuguilla fue de 3.25 y el de la yuca de 3.00 , lo que confirmó que ambos materiales son de carácter ácido y la diferencia entre estos valores se atribuyó a las variaciones de la concentración de sitios ácidos totales presentes en la superficie de los bioadsorbentes. Además, los valores estimados de PCC son similares a los valores que han sido reportados por otros autores para distintos materiales bioadsorbentes (Hamissa et al. 2010, Asberry et al. 2014).

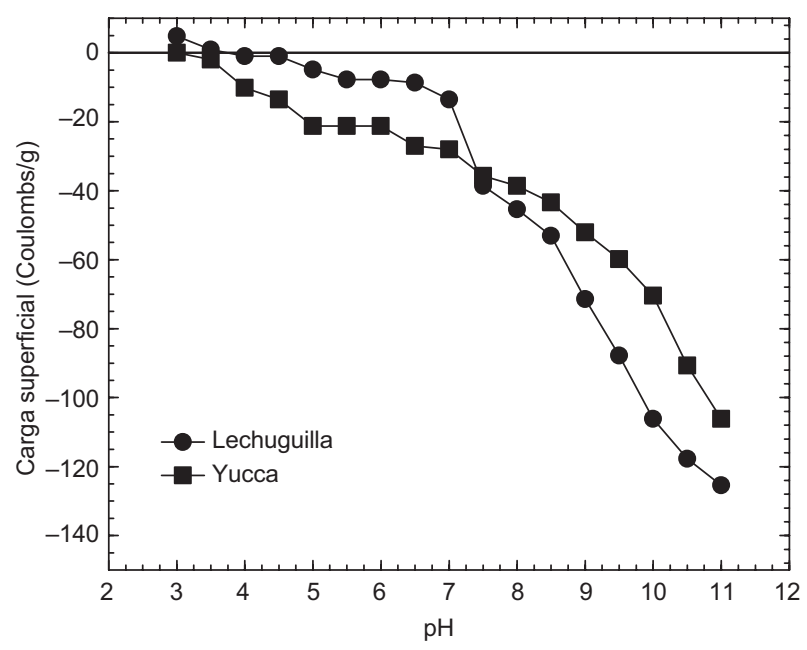

Fig. 1. Distribución de la carga superficial y punto de carga cero (PCC) de las muestras de lechuguilla y yuca

El análisis de adsorción física evidenció que estos materiales tienen una baja porosidad y por lo tanto poseen un área específica muy pequeña, lo cual es característico de los bioadsorbentes (Cuadro I). También, se determinó el contenido de cenizas y humedad de estas fibras naturales cuyos valores no se han reportado en la literatura (Cuadro I). El contenido de cenizas y humedad en materiales bioadsorbentes es muy variable (Wan y Hanafiah 2008, Velazquez et al. 2013, Salazar y Leyva 2016) y se asocia con la cantidad de micronutrientes inorgánicos y las características hidrofílicas o hidrofóbicas del material, respectivamente. Asimismo, la cantidad de agua en fibras naturales se asocia con la capacidad de hinchamiento del material (Demirbas 2008). El contenido de cenizas y humedad cuantificado en este estudio fue inferior a los de la celulosa, lignina y hemicelulosa reportados en fibras naturales (Vieira et al. 2002), por lo que no se consideró que tuvieran una contribución importante a la bioadsorción de $\mathrm{Pb}$ (II) como resultó ser la de estos constituyentes orgánicos. 

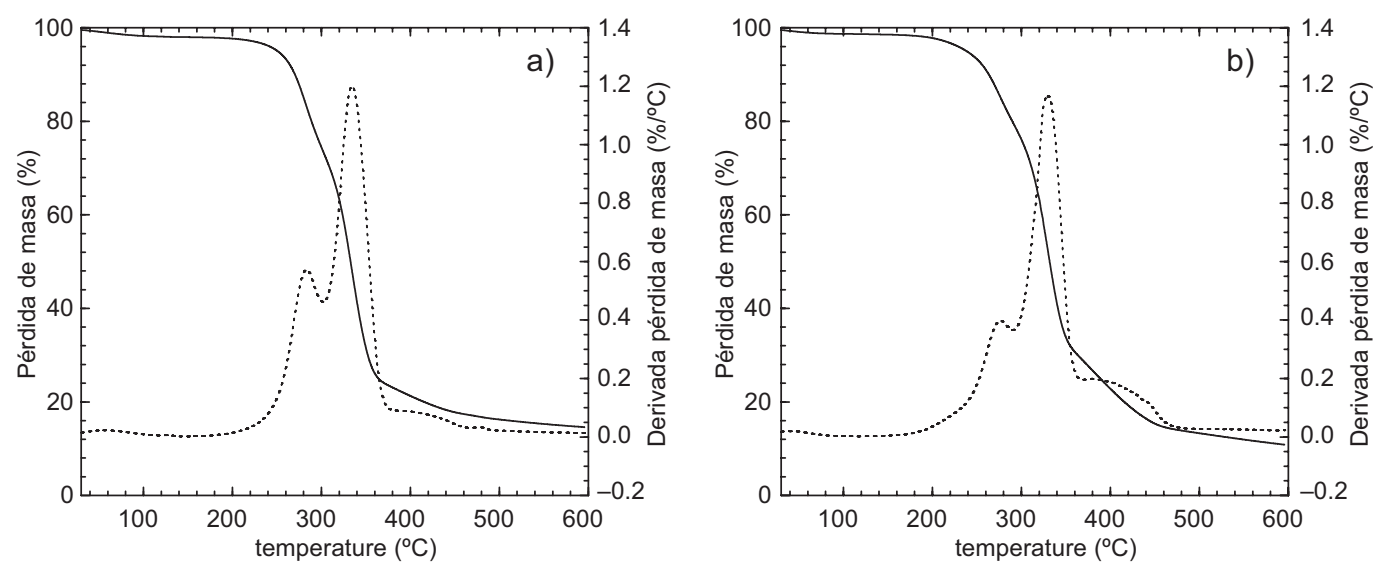

Fig. 2. Análisis termogravimétrico y diferencial de los dos materiales bioadsorbentes: a) lechuguilla y b) yuca

En las figuras $\mathbf{2 a}$ y $\mathbf{2 b}$ se ilustran los termogramas de la descomposición de la lechuguilla y yuca, respectivamente. En ambos casos, es posible apreciar tres regiones características en la descomposición de fibras vegetales que corresponden a la degradación de hemicelulosa, celulosa y lignina, en los intervalos de 200 a $330{ }^{\circ} \mathrm{C}, 200$ a $450{ }^{\circ} \mathrm{C}$ y 400 a $600{ }^{\circ} \mathrm{C}$, respectivamente (Conesa 2000). La descomposición de la celulosa en ambos materiales constituye el principal proceso de degradación, lo cual era de esperarse, pues esta macromolécula es el principal componente de las fibras vegetales y se estimaron pérdidas de peso del 50.3 y $47.5 \%$ p/p para la lechuguilla y la yuca, respectivamente. Respecto a la hemicelulosa, fueron del 20.7 y $17.2 \%$ p/p y para la lignina fueron del 9.6 y $17.7 \%$ p/p. Con base en esta información se concluyó que la yuca es un material con un mayor contenido de lignina.

Los espectros en el infrarrojo de las muestras de la lechuguilla y la yuca sin saturar y saturadas con $\mathrm{Pb}$ (II) se muestran en la figura 3. En los espectros de las muestras sobresalen las bandas asignadas a los grupos O-H (región de 3400 a $3100 \mathrm{~cm}^{-1}$ ), C-H (3000 a $\left.2700 \mathrm{~cm}^{-1}\right), \mathrm{C}-\mathrm{O}\left(1320\right.$ a $\left.1210 \mathrm{~cm}^{-1}\right)$ y C $=\mathrm{O}$ (1730-1700 $\left.\mathrm{cm}^{-1}\right)$. La conjugación de estas bandas indica la naturaleza biopolimérica de ambos materiales y la banda de baja intensidad a $1730 \mathrm{~cm}^{-1}$ la presencia de grupos carboxílicos en la superficie en concentraciones mínimas, lo que coincide con los valores mostrados en el cuadro I (Pavia et al. 2015). Con la finalidad de evidenciar las interacciones entre el $\mathrm{Pb}$ (II) y los grupos funcionales de la superficie de los materiales, se procedió a realizar un análisis FTIR a los bioadsorbentes saturados con el bioadsorbato. En el caso de la yuca, se observó una leve atenuación de la banda del grupo carbonilo $\left(1730 \mathrm{~cm}^{-1}\right)$ con respecto a la banda del grupo amida $\left(1655 \mathrm{~cm}^{-1}\right)$,

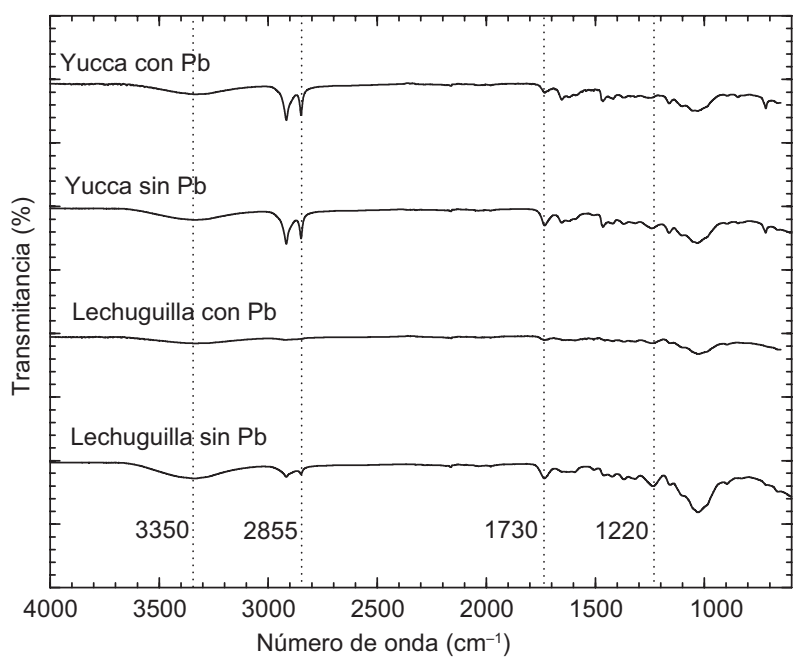

Fig. 3. Espectros en el infrarrojo de las muestras de lechuguilla y yuca sin saturar y saturados con plomo

corroborando con ello las interacciones entre el grupo carboxilo y el plomo. Por otra parte, en el caso de la muestra de lechuguilla saturada con $\mathrm{Pb}$ (II) se apreció una atenuación general de todo el espectro. Esto, más que indicar interacción con algún grupo funcional específico, indica el recubrimiento de la muestra por algún precipitado.

En la figuras 4a y 4c se ilustran imágenes del ESEM de las muestras de lechuguilla y yuca, respectivamente. En ellas se observa la textura de las muestras y se corrobora su naturaleza fibrosa, propia de los materiales lignocelulósicos los cuales están conformados por filamentos. Por otra parte, las figuras $\mathbf{4 b}$ y 4d ilustran las imágenes del ESEM de las muestras de lechuguilla y yuca con $\mathrm{Pb}$ (II) bioadsorbido, respectivamente. En el caso de la muestra de lechuguilla es posible apreciar micropartículas aglomeradas en la superficie del material, lo que indica un mecanismo 


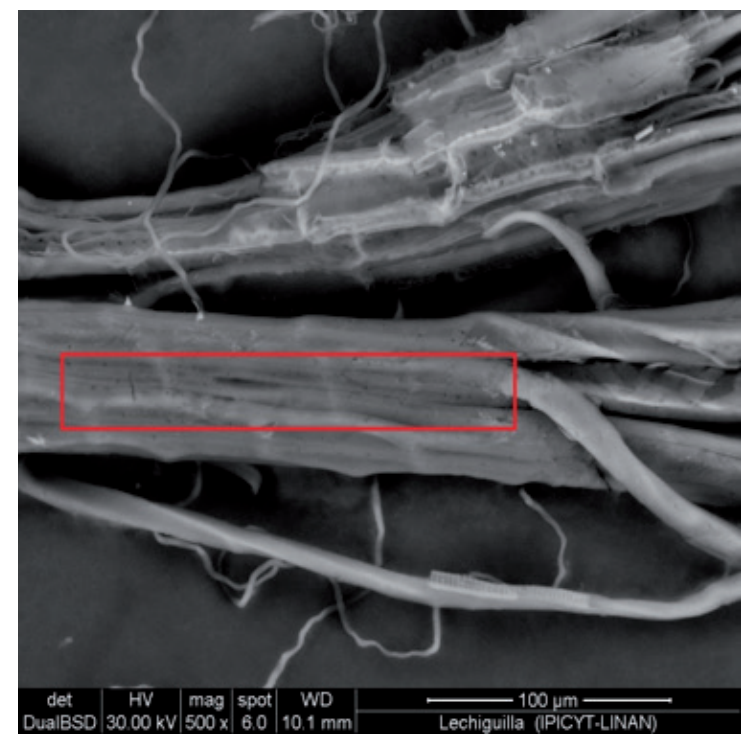

a)

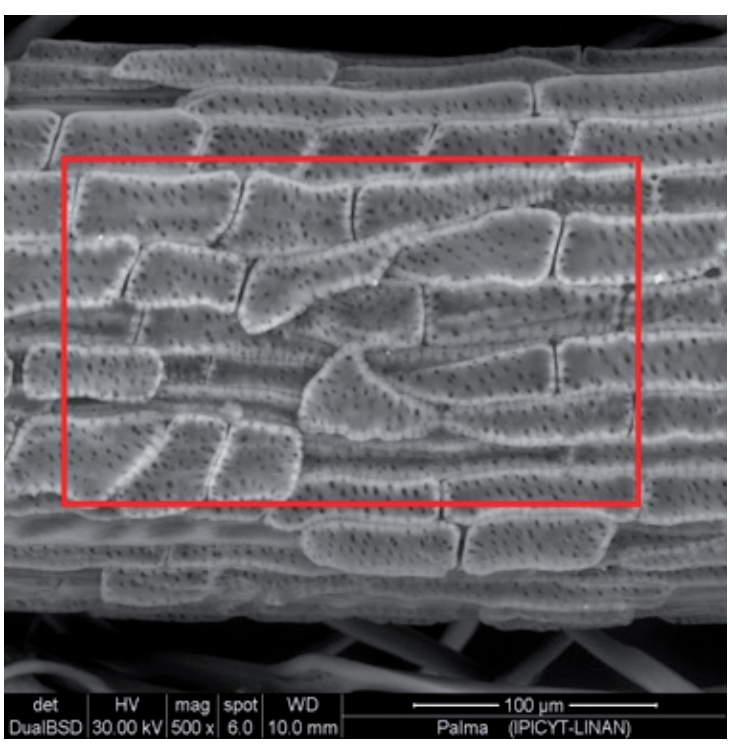

c)

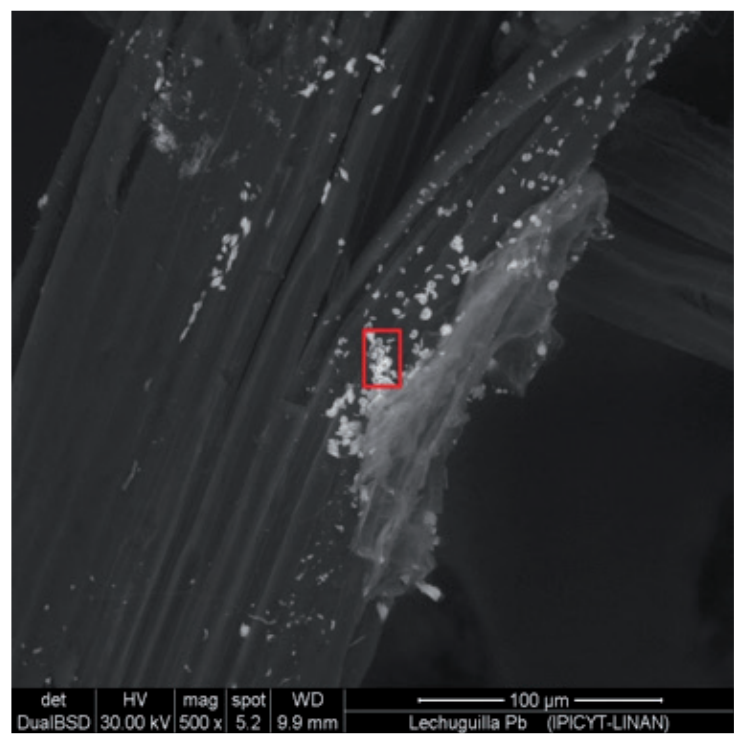

b)

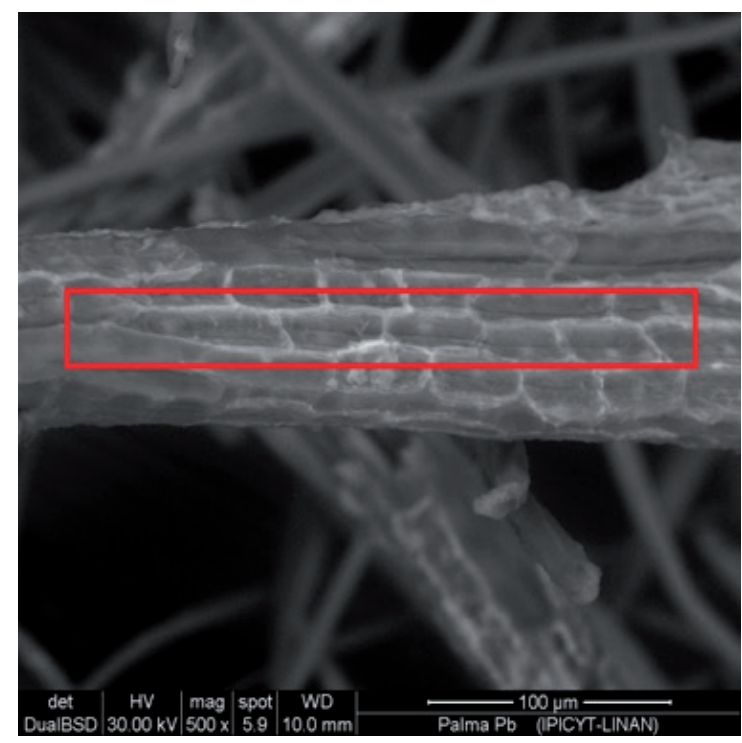

d)

Fig. 4. Imágenes del microscopio electrónico de barrido ambiental de los bioadsorbentes sin saturar y saturados con $\mathrm{Pb}$ (II): a) lechuguilla antes de la bioadsorción $500 \times$, b) lechuguilla después de la bioadsorción $500 \times$, c) yuca antes de la bioadsorción $500 \times$ y d) yuca después de la bioadsorción $500 \times$

de microprecipitación; mientras que la muestra de yuca no presentó microprecipitados, sino que el plomo se bioadsorbió en toda la superficie. La presencia del plomo en la superficie de los materiales se confirmó por medio del análisis elemental realizado por espectroscopía de energía dispersiva de rayos-X (EDS) acoplada al ESEM. Los espectrogramas de energía dispersiva de cada muestra fueron obtenidos de las zonas señaladas en la figura 4 y se ilustran en la figura 5. Aunque el área de análisis por EDS en la muestra de yuca fue mayor que la de la lechuguilla, la intensidad de la señal de $\mathrm{Pb}$ (II) detectada con respecto al pico del carbón fue menor y esto se asocia a la microprecipitación de $\mathrm{Pb}$ (II) que ocurre sobre la muestra de lechuguilla y que se relaciona con la atenuación general de su espectro FTIR (Fig. 3). Lo anterior revela que el $\mathrm{Pb}$ (II) se remueve por distintos mecanismos en ambos materiales.

\section{Isotermas de bioadsorción}

Los modelos de las isotermas de Freundlich y Langmuir se emplearon para ajustar los datos 

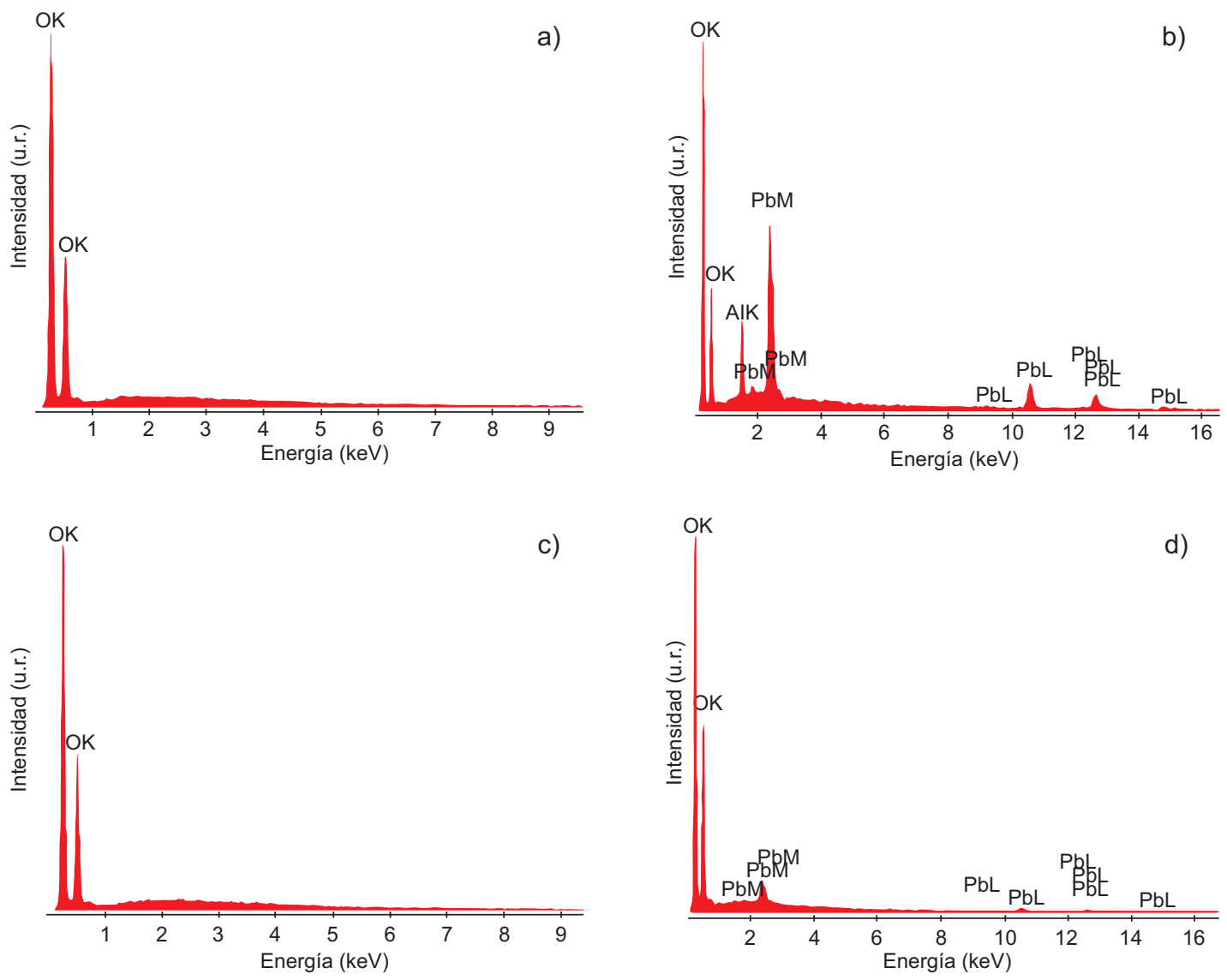

Fig. 5. Espectrogramas de energía dispersiva de rayos-X de los bioadsorbentes sin saturar y saturados con $\mathrm{Pb}$ (II): a) lechuguilla antes de la bioadsorción, b) lechuguilla después de la bioadsorción, c) yuca antes de la bioadsorción y d) yuca después de la bioadsorción

experimentales del equilibrio de bioadsorción de $\mathrm{Pb}$ (II) en solución acuosa sobre lechuguilla y yuca. Estos modelos se representan matemáticamente por las ecuaciones 2 y 3 , las cuales se muestran a continuación:

$q=\frac{q_{m} K_{L} C}{1+K_{L} C}$

$\mathrm{q}=\mathrm{k}_{\mathrm{F}} \mathrm{C}^{1 / \mathrm{n}}$

donde $\mathrm{C}(\mathrm{mg} / \mathrm{L})$ es la concentración de $\mathrm{Pb}$ (II) en el equilibrio, $\mathrm{K}_{\mathrm{L}}(\mathrm{L} / \mathrm{mg})$ y $\mathrm{q}_{\mathrm{m}}(\mathrm{mg} / \mathrm{g})$ son las constantes de Langmuir relacionadas con la energía libre de bioadsorción y la máxima capacidad de bioadsorción, respectivamente, $\mathrm{k}_{\mathrm{F}}\left(\mathrm{L}^{1 / n} \mathrm{mg}^{1-1 / n} \mathrm{~g}^{-1}\right)$ y $1 / \mathrm{n}$ son las constantes de Freundlich relacionadas con la capacidad de bioadsorción y la intensidad, respectivamente y q (mg/g) es la capacidad de bioadsorción de $\mathrm{Pb}$ (II) del bioadsorbente.
Las constantes para estas isotermas fueron evaluadas por un método de mínimos cuadrados basado en un algoritmo de optimización y se muestran en el cuadro II, así como el porcentaje de desviación promedio, \% Desv., el cual se define como:

$\%$ Desv. $=\sum_{\mathrm{i}=1}^{\mathrm{N}}\left|\frac{\mathrm{q}_{\exp }-\mathrm{q}_{\mathrm{pred}}}{\mathrm{q}_{\exp }}\right| \times 100 \%$

donde $\mathrm{N}$ es el número de datos experimentales, $\mathrm{q}_{\exp }$ (mg/g) es la capacidad de bioadsorción de $\mathrm{Pb}$ (II) experimental obtenida con la ecuación 1 y $q_{\text {pred }}(\mathrm{mg} / \mathrm{g})$ es la capacidad de bioadsorción de $\mathrm{Pb}$ (II) predicha con los modelos de isotermas.

Se consideró que el modelo que mejor interpretó los datos experimentales fue aquel que presentó el menor porcentaje de desviación. Bajo este criterio, el modelo de Langmuir ajustó mejor 9 de los 10 casos de estudio, así que con este modelo se decidió interpretar los datos del equilibrio de bioadsorción de $\mathrm{Pb}$ (II) sobre ambos materiales. 
CUADRO II. PARÁMETROS Y PORCENTAJE DE DESVIACIÓN DE LOS MODELOS DE LAS ISOTERMAS DE LANGMUIR Y FREUNDLICH

\begin{tabular}{|c|c|c|c|c|c|c|c|c|}
\hline \multirow{2}{*}{ Bioadsorbente } & \multirow{2}{*}{$\mathrm{pH}$} & \multicolumn{4}{|c|}{ Langmuir } & \multicolumn{3}{|c|}{ Freundlich } \\
\hline & & $\mathrm{T}\left({ }^{\circ} \mathrm{C}\right)$ & $\mathrm{q}_{\mathrm{m}}$ & $\mathrm{K}_{\mathrm{L}}$ & \% Desv. & $\mathrm{k}_{\mathrm{F}}$ & $1 / \mathrm{n}$ & $\%$ Desv \\
\hline \multirow{5}{*}{ Lechuguilla } & 2 & 25 & 1.093 & 0.050 & 8.331 & 0.219 & 0.295 & 10.98 \\
\hline & 3 & 25 & 4.110 & 0.029 & 4.073 & 0.622 & 0.325 & 8.149 \\
\hline & 5 & 25 & 13.49 & 0.030 & 7.743 & 2.451 & 0.289 & 9.911 \\
\hline & 5 & 15 & 7.856 & 0.028 & 3.097 & 1.151 & 0.332 & 5.690 \\
\hline & 5 & 35 & 19.55 & 0.031 & 6.121 & 2.834 & 0.329 & 9.527 \\
\hline \multirow{5}{*}{ Yuca } & 2 & 25 & 5.076 & 0.020 & 8.050 & 0.756 & 0.311 & 14.07 \\
\hline & 3 & 25 & 8.261 & 0.129 & 3.073 & 3.031 & 0.177 & 12.64 \\
\hline & 5 & 25 & 20.45 & 0.190 & 13.00 & 7.128 & 0.207 & 2.400 \\
\hline & 5 & 15 & 15.85 & 0.164 & 6.421 & 4.829 & 0.228 & 18.04 \\
\hline & 5 & 35 & 24.12 & 0.205 & 16.98 & 6.108 & 0.287 & 8.79 \\
\hline
\end{tabular}

$\mathrm{q}_{\mathrm{m}}=$ máxima capacidad de bioadsorción, $\mathrm{K}_{\mathrm{L}}=$ constante de la isoterma de Langmuir relacionada con la energía libre de bioadsorción, $\mathrm{k}_{\mathrm{F}}=$ constante de la isoterma de Freundlich relacionada con la capacidad de bioadsorción, $1 / \mathrm{n}=$ constante de la isoterma de Freundlich relacionada con la intensidad de la bioadsorción

\section{Efecto del pH en la isoterma de bioadsorción de Pb (II)}

En las figuras 6a y $\mathbf{6 b}$ se observa el efecto del $\mathrm{pH}$ de la solución sobre la capacidad de bioadsorción de $\mathrm{Pb}$ (II) de la lechuguilla y yuca, respectivamente. Para ambos casos se observó que al incrementar el $\mathrm{pH}$ de la solución de 2.0 a 5.0 la capacidad de bioadsorción se incrementa considerablemente. En el caso de la lechuguilla, la capacidad máxima de bioadsorción de $\mathrm{Pb}$ (II) se incrementó 12.34 y 3.28 veces al aumentar el $\mathrm{pH}$ de la solución de 2.0 a 5.0 y de 3.0 a 5.0, respectivamente; mientras que para la yuca aumentó 4.03 y 2.48 veces para los mismos intervalos de $\mathrm{pH}$. El efecto significativo del $\mathrm{pH}$ sobre la isoterma de bioadsorción se atribuye a las interacciones electrostáticas entre el $\mathrm{Pb}$ (II) en solución y la superficie de los bioadsorbentes. Como se ilustra en la figura 1, conforme el $\mathrm{pH}$ de la solución sea más elevado, la carga neta negativa de los materiales se va incrementando, lo que propiciará una mayor atracción entre los cationes metálicos presentes en solución y la superficie del bioadsorbente. Este comportamiento se ha observado para la remoción de $\mathrm{Pb}$ (II) empleando otros bioadsorbentes como Agave americana y cáscara de cacahuate (Hamissa et al. 2010, Taşar et al. 2014).

El intercambio iónico es otro mecanismo que se puede presentar en la bioadsorción de $\mathrm{Pb}$ (II) sobre la lechuguilla y la yuca y esto se validó al observar que durante la bioadsorción, el $\mathrm{pH}$ de la solución siempre disminuyó y para mantenerlo constante fue necesario añadir algunas gotas de las soluciones $0.01 \mathrm{~N}$ de $\mathrm{NaOH}$ o $\mathrm{HNO}_{3}$. En los experimentos realizados a $\mathrm{pH}$ de 3.0 y 5.0, el pH de la solución disminuyó considerablemente el primer día (entre 0.4 y 0.9 unidades)
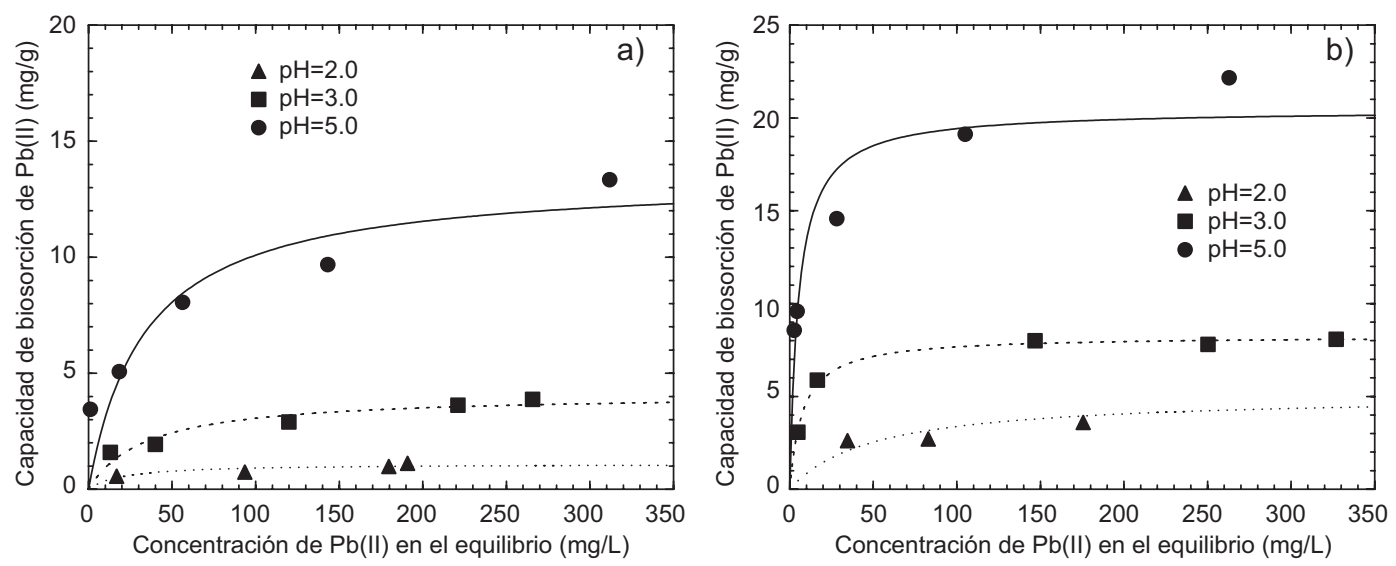

Fig. 6. Efecto del $\mathrm{pH}$ de la solución sobre la capacidad de bioadsorción de los biomateriales a $\mathrm{T}=25^{\circ} \mathrm{C}$. Las líneas representan el modelo de la isoterma de Langmuir: a) lechuguilla y b) yuca 

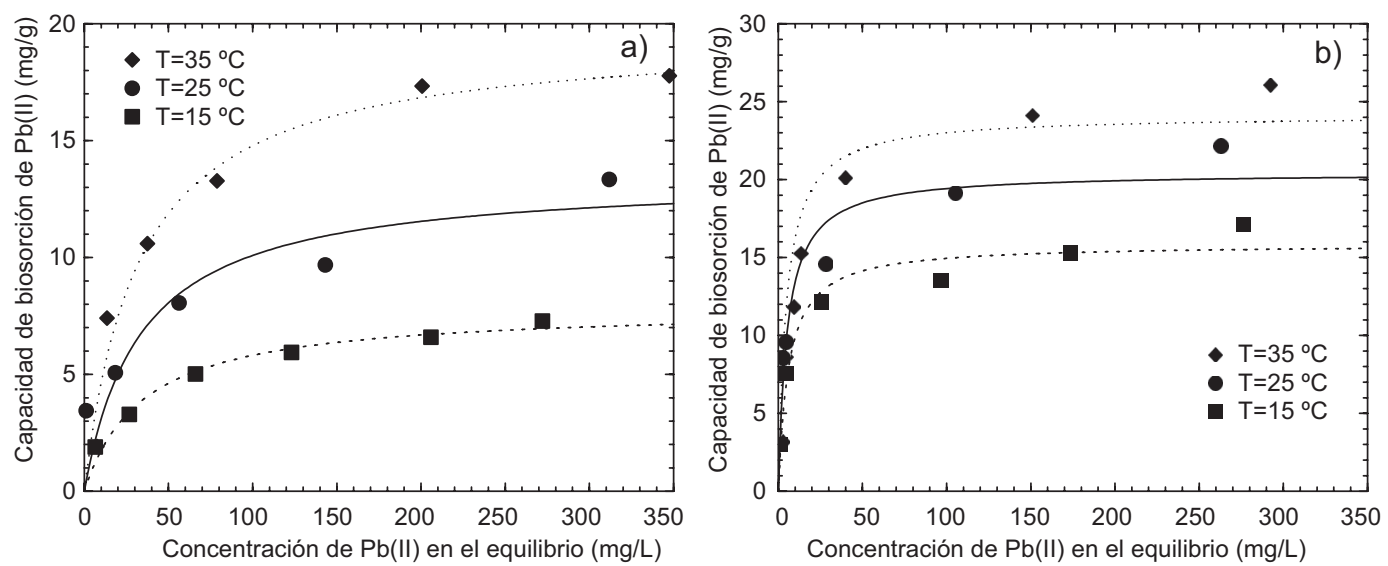

Fig. 7. Efecto de la temperatura de la solución sobre la capacidad de bioadsorción de los biomateriales a $\mathrm{pH}=$ 5.0. Las líneas representan el modelo de la isoterma de Langmuir: a) lechuguilla y b) yuca

mientras que en los experimentos a $\mathrm{pH}$ de 2.0 difícilmente varió, es decir, el pH permaneció constante. Esto indica que los cationes $\mathrm{H}^{+}$se transfirieron de la superficie de los bioadsorbentes a la solución y los iones $\mathrm{Pb}$ (II) se transfirieron en dirección opuesta.

\section{Efecto de la temperatura en la isoterma de bio- adsorción de $\mathbf{P b}$ (II)}

Los resultados del efecto de la temperatura de la solución sobre la capacidad de bioadsorción de $\mathrm{Pb}$ (II), se ilustran en las figuras $7 \mathbf{a}$ y $7 \mathbf{b}$ para la lechuguilla y la yuca, respectivamente. El intervalo de temperaturas estudiado fue de 15 a $35^{\circ} \mathrm{C}$ a un $\mathrm{pH}$ $=5.0$. Los resultados revelaron que la capacidad de bioadsorción de $\mathrm{Pb}$ (II) de ambos materiales aumenta al incrementarse la temperatura de la solución. Las capacidades máximas de bioadsorción de $\mathrm{Pb}$ (II) de la lechuguilla y la yuca se incrementaron 1.72 y 1.45 veces y 1.29 y 1.18 veces al aumentar la temperatura de 15 a $25^{\circ} \mathrm{C}$ y de 25 a $35^{\circ} \mathrm{C}$, respectivamente. Esto indica que la bioadsorción de $\mathrm{Pb}$ (II) sobre la lechuguilla y la yuca es un proceso endotérmico.

\section{Parámetros termodinámicos de la bioadsorción de $\mathrm{Pb}$ (II)}

En el modelo de la isoterma de Langmuir, el parámetro $\mathrm{K}_{\mathrm{L}}$ es dependiente de la temperatura y puede emplearse para predecir parámetros termodinámicos como la energía libre de Gibss $\left(\Delta \mathrm{G}^{\circ}\right)$, la entalpía $\left(\Delta \mathrm{H}^{\circ}\right)$ y la entropía $\left(\Delta \mathrm{S}^{\circ}\right)$ a través de las siguientes ecuaciones:

$\Delta \mathrm{G}^{\circ}=-\mathrm{RT} \operatorname{Ln} \mathrm{K}_{\mathrm{L}}$

$\operatorname{Ln} K_{L}=-\frac{\Delta \mathrm{G}^{\circ}}{\mathrm{RT}}=-\frac{\Delta \mathrm{H}}{\mathrm{RT}}+\frac{\Delta \mathrm{S}^{\circ}}{\mathrm{R}}$
Los parámetros termodinámicos $\Delta \mathrm{H}^{\circ}$ y $\Delta \mathrm{S}^{\circ}$ se estimaron a partir de una regresión lineal de los datos del gráfico de $\mathrm{Ln} \mathrm{K}_{\mathrm{L}}$ contra $1 / \mathrm{T}$ (Fig. 8) y con la ecuación 5 se calcularon los valores de $\Delta \mathrm{G}^{\circ}$. Los parámetros termodinámicos evaluados para el proceso de bioadsorción de $\mathrm{Pb}$ (II) sobre los bioadsorbentes se muestran en el cuadro III. Los valores negativos de $\Delta \mathrm{G}^{\circ}$ a las temperaturas estudiadas indican que la bioadsorción de $\mathrm{Pb}$ (II) sobre la lechuguilla y la yuca es espontánea. En el estudio del efecto de la temperatura en la isoterma de bioadsorción de $\mathrm{Pb}$ (II) se determinó que la bioadsorción de $\mathrm{Pb}$ (II) sobre estos materiales es endotérmica. Los valores positivos de $\Delta \mathrm{H}^{\circ}$ estimados corroboran la naturaleza endotérmica de la bioadsorción de $\mathrm{Pb}$ (II) de ambos materiales. Los valores positivos de

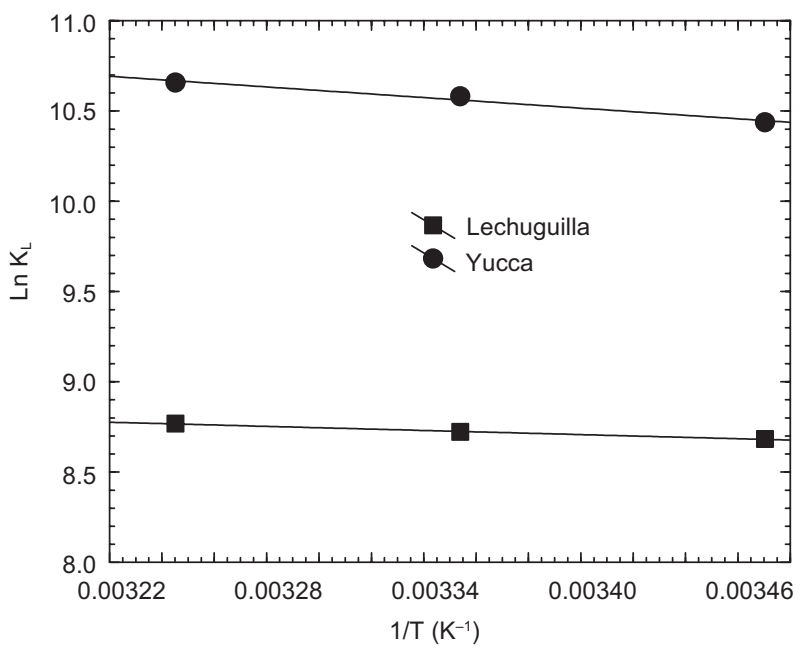

Fig. 8. Estimación de los parámetros termodinámicos de la bioadsorción de $\mathrm{Pb}$ (II) sobre las muestras de lechuguilla y yuca 
CUADRO III. PARÁMETROS TERMODINÁMICOS ESTIMADOS A PARTIR DE LA CONSTANTE DE LANGMUIR $\left(\mathrm{K}_{\mathrm{L}}\right)$ PARA LA BIOADSORCIÓN DE Pb (II) SOBRE LECHUGUILLA Y YUCA

\begin{tabular}{lcccccc}
\hline Bioadsorbente & $\mathrm{T}\left({ }^{\circ} \mathrm{C}\right)$ & $\begin{array}{c}\mathrm{K}_{\mathrm{L}} \\
(\mathrm{L} / \mathrm{mg})\end{array}$ & $\begin{array}{c}\mathrm{K}_{\mathrm{L}} \times 10^{-3} \\
(\mathrm{~L} / \mathrm{mol})\end{array}$ & $\begin{array}{c}\Delta \mathrm{G}^{\circ} \\
(\mathrm{kJ} / \mathrm{mol})\end{array}$ & $\begin{array}{c}\Delta \mathrm{H}^{\circ} \\
(\mathrm{kJ} / \mathrm{mol})\end{array}$ & $\begin{array}{c}\Delta \mathrm{S}^{\circ} \\
(\mathrm{J} / \mathrm{mol} \mathrm{K})\end{array}$ \\
\hline \multirow{3}{*}{ Lechuguilla } & 15 & 0.028 & 5.8948 & -20.80 & & \\
& 25 & 0.030 & 6.1424 & -21.62 & 3.166 & 83.16 \\
& 35 & 0.031 & 6.4232 & -22.46 & & \\
Yuca & 15 & 0.164 & 34.081 & -25.00 & & \multirow{2}{*}{115.1} \\
& 25 & 0.190 & 39.413 & -26.23 & 8.154 & \\
\hline
\end{tabular}

$\mathrm{K}_{\mathrm{L}}=$ constante de la isoterma de Langmuir relacionada con la energía libre de bioadsorción, $\mathrm{DG}^{\circ}=$ energía libre Gibss, $\mathrm{DH}^{\circ}=$ entalpía, $\mathrm{DS}^{\circ}=$ entropía

$\Delta \mathrm{S}^{\circ}$ muestran la afinidad del $\mathrm{Pb}$ (II) en solución por los bioadsorbentes. Los valores de los parámetros termodinámicos del equilibrio de bioadsorción de $\mathrm{Pb}$ (II) sobre la yuca fueron mayores que los obtenidos para la lechuguilla, lo cual indica que existe una mayor afinidad del $\mathrm{Pb}$ (II) por la yuca y por ello las capacidades de bioadsorción de este metal, a los distintos $\mathrm{pH}$ y temperaturas de solución, son mayores que las de la lechuguilla y esto se atribuyó al mayor contenido de sitios ácidos totales en este material.

\section{Mecanismo de bioadsorción}

La bioadsorción de metales es un proceso complejo afectado por diversos factores. Los mecanismos involucrados incluyen la adsorción química, la complejación, la adsorción sobre la superficie y poros, el intercambio iónico, la microprecipitación, la adsorción por fuerzas físicas y la condensación de hidróxidos metálicos sobre la superficie, entre otros (Demirbas 2008, Sud et al. 2008). Por lo anterior, es difícil cuantificar la presencia o contribución de cada posible mecanismo. Sin embargo, en este caso, para la remoción de $\mathrm{Pb}$ (II) sobre los bioadsorbentes estudiados se encontró que el aumento del $\mathrm{pH}$ de la solución favorece el incremento en la densidad de la carga superficial negativa, según lo ilustra la información presentada en el cuadro II y la figura 1, indicando con ello que un mecanismo de adsorción por fuerzas físicas o atracción electrostática entre la superficie de los materiales y los iones $\mathrm{Pb}$ (II) se lleva a cabo. De igual forma, las variaciones del $\mathrm{pH}$ de la solución durante las pruebas de bioadsorción de $\mathrm{Pb}$ (II) en ambos materiales, revelaron que el intercambio iónico se lleva a cabo, ya que los cationes $\mathrm{H}^{+}$se transfieren de la superficie de los bioadsorbentes a la solución y los iones $\mathrm{Pb}$ (II) en dirección opuesta. No obstante, las atracciones electrostáticas y el intercambio iónico no son el único mecanismo que ocurre, ya que a $\mathrm{pH}=2.0$ la superficie de estos materiales presenta carga positiva y aún cuando las atracciones electrostáticas serían repulsivas, se determinaron capacidades máximas de bioadsorción de 1.09 y $5.08 \mathrm{mg} / \mathrm{g}$ para la lechuguilla y la yuca, respectivamente. Un posible mecanismo que explicaría este fenómeno es la adsorción química, sin embargo; queda descartado ya que en ambos materiales el calor de bioadsorción tiene valores muy inferiores a $42 \mathrm{~kJ} / \mathrm{mol}$, que es el valor mínimo de energía que requiere este proceso (Do 1998). Un mecanismo factible para estas condiciones de operación son las interacciones $\pi$-catión, las cuales se presentan entre la nube electrónica de un anillo aromático y los cationes en solución (Gallivan y Dougherty 1999, Rivera y Sánchez 2003). La lignina presenta en su estructura molecular anillos aromáticos que propiciarían este tipo de interacciones. A través de los termogramas (Fig. 2) se determinó que la yuca presenta una mayor proporción de lignina que la lechuguilla, por tanto existe una mayor concentración de estructuras aromáticas y por ende se favorece en mayor proporción dichas interacciones, lo que explica porque a $\mathrm{pH}=$ 2.0 la yuca remueve más $\mathrm{Pb}$ (II) que la lechuguilla. Finalmente, la información proporcionada por las imágenes del ESEM de las muestras saturadas con $\mathrm{Pb}$ (II) a $\mathrm{pH}=5.0$ (Fig. 4), reveló que el $\mathrm{Pb}$ (II) se bioadsorbe sobre toda la superficie de la muestra de yuca, lo que es acorde con un mecanismo de atracción electrostática. Sin embargo, en el caso de la muestra de lechuguilla se observaron aglomeraciones de $\mathrm{Pb}$ (II) que pueden atribuirse a la formación de complejos o quelatos microprecipitados sobre la muestra. En estudios preliminares se ha reportado que la lechuguilla posee agentes quelantes naturales como son la yucagenina y el ruizgenin, que pueden ser usados en la remoción de metales pesados $(\mathrm{Cr}, \mathrm{Cd}, \mathrm{Cu}, \mathrm{Pb}$ y $\mathrm{Zn})$ de diversos ambientes (Romero et al. 2005). 
Con base en lo anterior, se consideró que la bioadsorción de $\mathrm{Pb}$ (II) en ambos materiales ocurre por los mecanismos de interacciones $\pi$-catión, atracciones electrostáticas e intercambio iónico, además en el caso de la lechuguilla se evidenció un proceso de microprecipitación, que no fue identificado para la yuca.

En diversos estudios sobre la bioadsorción de metales pesados en materiales lignocelulósicos se ha demostrado que la bioadsorción de estos contaminantes es reversible si se trata la biomasa con soluciones ácidas (Vaghetti et al. 2009, Velazquez et al. 2013, Salazar y Leyva 2016) o alcalinas (Agarwal et al. 2006). Sin embargo, si la bioadsorción se da por interacciones químicas, la desadorción será disminuida por la fuerza de los enlaces químicos presentes en la superficie del biomaterial (Agarwal et al. 2006). La regeneración de los bioadsorbentes es importante sobre todo si la preparación o generación de la biomasa es costosa. Para el caso de los residuos industriales empleados en este estudio, es de esperarse que la regeneración sea factible con soluciones ácidas o básicas pero en distinto grado. Siendo menos factible la regeneración de la lechuguilla por el mecanismo de microprecipitación que presenta.

\section{CONCLUSIONES}

La capacidad para bioadsorber $\mathrm{Pb}$ (II) es mayor en la yuca y esto se atribuyó principalmente a la diferencia en el contenido de sitios ácidos y lignina y el tipo de mecanismo de bioadsorción. En ambos materiales la bioadsorción de $\mathrm{Pb}$ (II) ocurre por los mecanismos de interacciones $\pi$-catión, atracciones electrostáticas e intercambio iónico, además, en el caso de la lechuguilla existe un proceso de microprecipitación.

Finalmente, se concluye que los residuos de las fibras naturales Agave lechuguilla Torr. (lechuguilla) y Yucca carnerosana (Trel.) McKelvey (yuca) son una alternativa para la bioadsorción de $\mathrm{Pb}$ (II) de soluciones acuosas.

\section{REFERENCIAS}

Agarwal G.S., Bhuptawat H.K. y Chaudhari S. (2006). Biosorption of aqueous chromium (VI) by Tamarindus indica seeds. Biores. Technol. 97 (7), 949-956.

DOI: $10.1016 /$ j.biortech.2005.04.030

AOAC (1990). Official methods of analysis of the Association of Official Analytical Chemists. Washington, D.C., EUA, 1230 pp.
Asberry H.B., Kuo C-Y., Gung C-H. y Suen S-Y. (2014). Characterization of water bamboo husk biosorbents and their application in heavy metal ion trapping. Microchem. J. 113, 59-63.

DOI: 10.1016/j.microc.2013.11.011

Blázquez G., Calero M., Hernáinz F., Tenorio G. y Martín M.A. (2010). Equilibrium biosorption of lead(II) from aqueous solution by solid waste from olive-oil production. Chem. Eng. J. 160 (2), 615-622.

DOI: $10.1016 /$ j.cej.2010.03.085

Boehm H.P. (1994). Some aspects of the surface chemistry of carbon blacks and other carbons. Carbon 32 (5), 759-769.

DOI: $10.1016 / 0008-6223(94) 90031-0$

Conesa A. (2000). Curso básico de análisis térmico. 1a ed. Club Universitario, Alicante, España, 105 pp.

Demirbas A. (2008). Heavy metal adsorption onto agrobased waste materials: A review. J. Hazard. Mater. 157 (2-3), 220-229. DOI: 10.1016/j.jhazmat.2008.01.024

Do D.D. (1998). Adsorption analysis: Equilibria and kinetics. 1a ed. Imperial College Press, Londres, Reino Unido, 892 pp.

Gallivan J.P. y Dougherty D.A. (1999). Cation- $\pi$ interactions in structural biology. Proc. Natl. Acad. Sci. USA 96 (17), 9459-9464. DOI: 10.1073/pnas.96.17.9459

Gupta S., Kumar D. y Gaur J.P. (2009). Kinetic and isotherm modeling of lead (II) sorption onto some waste plant materials. Chem. Eng. J. 148 (2-3), 226-233. DOI: $10.1016 /$ j.cej.2008.08.019

Hamissa A.M.B., Lodi A., Seffen M., Finocchio E., Botter R. y Converti A. (2010). Sorption of Cd (II) and Pb (II) from aqueous solutions onto Agave americana fibers. Chem. Eng. J. 159 (1-3), 67-74. DOI: $10.1016 / j$.cej.2010.02.036

O'Connell D.W., Birkinshaw C. y O'Dwyer T.F. (2008). Heavy metal adsorbents prepared from the modification of cellulose. Biores. Technol. 99 (15), 6709-6724. DOI: $10.1016 /$ j.biortech.2008.01.036

Pavia D.L., Lampman G.M., Kriz G.S. y Vyvyan J.A. (2015). Introduction to spectroscopy. 5a ed. Cengage Learning, California, EUA, 784 pp.

Reddad Z., Gerente C., Andres Y. y Le Cloirec P. (2002). Adsorption of several metal ions onto a low cost biosorbent: Kinetic and equilibrium studies. Environ. Sci. Technol. 36 (9), 2067-2073. DOI: $10.1021 /$ es0102989

Rivera J. y Sánchez M. (2003). Adsorption of Cr(III) on ozonised activated carbon. Importance of Cpi-cation interactions. Wat. Res. 37 (14), 3335-3340. DOI: $10.1016 / \mathrm{S} 0043-1354(03) 00177-5$

Romero J., Peralta J.R., Rodríguez E., Ramirez S.L. y Gardea J.L. (2005). Determination of thermodynamic parameters of $\mathrm{Cr}(\mathrm{VI})$ adsorption from aqueous solution 
onto Agave lechuguilla biomass. J. Chem. Thermodynamics 37 (4), 343-347.

DOI: $10.1016 /$ j.jct.2004.09.013

Romero J., Peralta J.R., Rodriguez E. Delgado M. y Gardea J.L. (2006). Potential of Agave lechuguilla biomass for $\mathrm{Cr}(\mathrm{III})$ removal from aqueous solutions: Thermodynamics studies. Biores. Technol. 97 (1), 178-182. DOI: 10.1016/j.biortech.2005.01.037

Romero J., Walton J.C., Peralta J.R., Rodríguez E., Romero J. y Gardea J.L. (2009). Modeling the adsorption of $\mathrm{Cr}$ (III) from aqueous solution onto Agave lechuguilla biomasss: Study of the advective and dispersive transport. J. Hazard. Mater. 161 (1), 360-365.

DOI: $10.1016 /$ j.jhazmat.2008.03.102

Romero J., García J.L., Velázquez G., Maldonado A.A., Canales I., Romero J. y Ramírez R. (2010). Modelación del proceso de transferencia de masa en columnas de lecho fijo de la bioadsorción del $\mathrm{Cr}(\mathrm{VI})$ usando transformadas de Laplace. CULCyT 41, 106-131.

Salazar J.J. y Leyva R. (2016). Novel biosorbent with high adsorption capacity prepared by chemical modification of white pine (Pinus durangensis) sawdust. Adsorption of $\mathrm{Pb}$ (II) from aqueous solutions. J. Environ. Manag. 169, 303-312. DOI: 10.1016/j.jenvman.2015.12.040

Sheldon S. (1980). Ethnobotany of Agave lecheguilla and Yucca carnerosana in Mexico's zona ixtlera. Econ. Bot. 34 (4), 376-390. DOI: 10.1007/BF02858314

Srivastava S., Agrawal S.B. y Mondal M.K. (2015). Biosorption isotherm and kinetics on removal of $\mathrm{Cr}(\mathrm{VI})$ using native and chemically modified Lagerstroemia speciosa bark. Ecol. Eng. 85, 56-66.

DOI: $10.1016 /$ j.ecoleng.2015.10.011

Sud D., Mahajan G. y Kaur M.P. (2008). Agricultural waste material as potential adsorbent for sequestering heavy metal ions from aqueous solution - A review. Biores. Technol. 99 (14), 6017-6027

DOI: 10.1016/j.biortech.2007.11.064

Taşar S., Kaya F. y Özer A. (2014). Biosorption of lead(II) ions from aqueous solution by peanut shells: Equilibrium thermodynamic and kinetic studies. J. Environ. Chem. Eng. 2 (2), 1018-1026.

DOI: $10.1016 /$ j.jece.2014.03.015

Velazquez L.H., Pavlick A. y Rangel J.R. (2013). Chemical characterization of raw and treated agave bagasse and its potential as adsorbent of metal cations from water. Ind. Crop. Prod. 43, 200-206. DOI: $10.1016 /$ j.indcrop.2012.06.049

Vieira M.C., Heinze Th., Cruz R. y Mendoza A.M. (2002). Cellulose derivatives from cellulosic material isolated from Agave lechuguilla and fourcroydes. Cellulose 9 (2), 203-212. DOI: 10.1023/A:1020158128506

Wan W.S. y Hanafiah M.A.K.M. (2008). Removal of heavy metal ions from wastewater by chemically modified plant wastes as adsorbents: A review. Biores. Technol. 99 (10), 3935-3948.

DOI: 10.1016/j.biortech.2007.06.011 